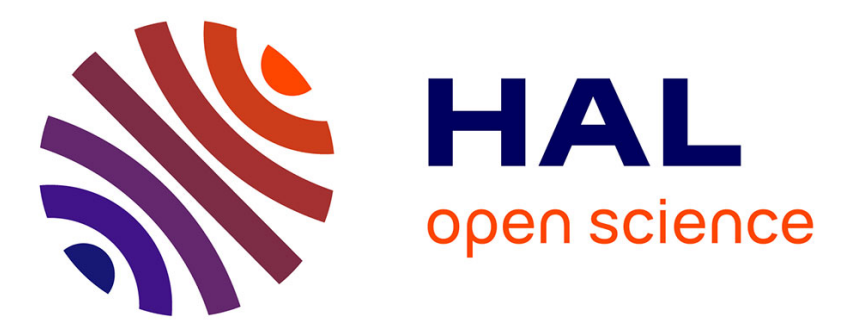

\title{
Phonon anomalies in pyrochlore iridates studied by Raman spectroscopy
}

K. Ueda, R. Kaneko, A. Subedi, M. Minola, B. Kim, J. Fujioka, Y. Tokura, B.

Keimer

\section{- To cite this version:}

K. Ueda, R. Kaneko, A. Subedi, M. Minola, B. Kim, et al.. Phonon anomalies in pyrochlore iridates studied by Raman spectroscopy. Physical Review B, 2019, 100 (11), 10.1103/physrevb.100.115157 . hal-03058678

\section{HAL Id: hal-03058678 \\ https://hal.science/hal-03058678}

Submitted on 12 Dec 2020

HAL is a multi-disciplinary open access archive for the deposit and dissemination of scientific research documents, whether they are published or not. The documents may come from teaching and research institutions in France or abroad, or from public or private research centers.
L'archive ouverte pluridisciplinaire HAL, est destinée au dépôt et à la diffusion de documents scientifiques de niveau recherche, publiés ou non, émanant des établissements d'enseignement et de recherche français ou étrangers, des laboratoires publics ou privés. 


\title{
Phonon anomalies in pyrochlore iridates studied by Raman spectroscopy
}

\author{
K. Ueda,${ }^{1}$ R. Kaneko, ${ }^{2}$ A. Subedi, ${ }^{3}$ M. Minola, ${ }^{1}$ B. J. Kim,${ }^{1,4}{ }^{4}$ J. Fujioka, ${ }^{6,7}$ Y. Tokura, ${ }^{2,8}$ and B. Keimer ${ }^{1}$ \\ ${ }^{1}$ Max-Planck-Institut für Festkörperforschung, Heisenbergstr. 1, D-70569 Stuttgart, Germany \\ ${ }^{2}$ Department of Applied Physics, University of Tokyo, Tokyo 113-8656, Japan \\ ${ }^{3}$ Centre de Physique Theorique, Ecole Polytechnique, CNRS, France \\ ${ }^{4}$ Department of Physics, Pohang University of Science and Technology, Pohang 790-784, Republic of Korea \\ ${ }^{5}$ Center for Artificial Low Dimensional Electonic Systems, \\ Institute for Basic Science (IBS), Republic of Korea \\ ${ }^{6}$ PRESTO, Japan Science and Technology Agency, Kawaguchi, Saitama 332-0012, Japan \\ ${ }^{7}$ University of Tsukuba, 1-1-1 Tennodai, Tsukuba, Ibaraki, 305-8573, Japan \\ ${ }^{8}$ Center for Emergent Matter Science (CEMS), RIKEN Advanced Science Institute (ASI), Wako 351-0198, Japan
}

(Dated: March 20, 2019)

\begin{abstract}
We report polarization-resolved Raman scattering measurements on single crystals of the pyrochlore compound $\mathrm{Eu}_{2} \mathrm{Ir}_{2} \mathrm{O}_{7}$ which exhibits a ground state with non-collinear magnetic order, as well as its paramagnetic counterpart $\operatorname{Pr}_{2} \mathrm{Ir}_{2} \mathrm{O}_{7}$. The spectra reveal the six Raman-active optical phonons expected for the pyrochlore lattice symmetry. Combined with density functional calculations, polarization analysis of the Raman intensity allows us to assign all observed peaks to specific vibration patterns. Whereas most phonon profiles are weakly temperature dependent, an Ir-O-Ir bond-bending vibration in $\mathrm{Eu}_{2} \mathrm{Ir}_{2} \mathrm{O}_{7}$ exhibits a pronounced Fano asymmetry in the paramagnetic state, and marked softening and lineshape anomalies at the magnetic phase transition. These observations indicate strong electron-phonon interactions, which must be considered in models of the electronic properties and phase behavior of the pyrochlore iridates. In addition, a prominent magnon mode appears in the Raman spectrum of $\mathrm{Eu}_{2} \mathrm{Ir}_{2} \mathrm{O}_{7}$ below the magnetic transition temperature.
\end{abstract}

Electronic correlations and topological phenomena are major themes of contemporary solid-state physics. Whereas research in both areas evolved from different origins, several emerging research frontiers now encompass both strong electronic correlations and topological complexity; prominent examples include topological superconductors and Kitaev spin liquids. [1] Materials with $4 d$ - and $5 d$-electrons are fertile platforms for exploration of these ideas, because the large spin-orbit coupling (SOC) of the valence electrons can both effectively enhance electronic correlations (by narrowing the valence band) [2] and generate topologically complex wavefunctions (by promoting spin-momentum locking). [3]

Iridates of composition $R_{2} \mathrm{Ir}_{2} \mathrm{O}_{7}$ (where $R$ is a rare earth element) have received particular recent attention in this regard. The $\mathrm{Ir}^{4+}$ ions with valence electron configuration $5 d^{5}$ and total angular momentum $J=\frac{1}{2}$ are arranged on the geometrically frustrated pyrochlore structure. Due to the confluence of strong SOC, strong Coulomb correlations, and geometric frustration, the pyrochlore iridates undergo multiple magnetic and metalinsulator transitions as a function of temperature, chemical composition [4-6], pressure [7-9], and magnetic field [10-13]. Figure 1 shows the phase diagram as a function of temperature and the radius of the $R$ ions, which modulates the valence-electron bandwidth and hence the effective correlation strength. Whereas compounds with large $R$ and large bandwidth are paramagnetic metals in the entire temperature range $[14,15]$, compounds with smaller $R$ exhibit a magnetically ordered state with sharply reduced electrical conductivity below a temperature $T_{N}$ that depends on $R$. The magnetic ordering pattern is the so-called "all-in/all-out" (AIAO) configuration with Ir magnetic moments pointing into and out of the tetrahedral units of the pyrochlore lattice in an alternating fashion [16-18]. For compounds with the smallest $R$, the electrical conductivity exhibits insulating behavior even in the paramagnetic state. The phase diagram is analogous to other systems with Mott metal-insulator transitions, [19] but the unusual electronic structure of $R_{2} \mathrm{Ir}_{2} \mathrm{O}_{7}$ as well as a host of anomalous thermodynamic, spectroscopic, and transport data have stimulated various proposals for topological phases and excitations [3]. What is missing to date is experimental insight into the role of lattice degrees of freedom. Theoretical work (see for instance Ref. [20]) suggests that the electron-lattice coupling plays a vital role in the determination of the ground states.

We have used polarization-resolved Raman scattering to measure the temperature evolution of phonon and magnon excitations in $R_{2} \mathrm{Ir}_{2} \mathrm{O}_{7}$ single crystals with $R=$ $\mathrm{Pr}$ and $\mathrm{Eu}$, which together cover all phases in the diagram of Fig. 1. Whereas $\mathrm{Pr}_{2} \mathrm{Ir}_{2} \mathrm{O}_{7}$ is a paramagnetic metal at all temperatures [15], $\mathrm{Eu}_{2} \mathrm{Ir}_{2} \mathrm{O}_{7}$ is insulating already in the paramagnetic state and exhibits AIAO order below $T_{N}=115 \mathrm{~K}$. [6] Since lattice distortions can relieve magnetic frustration, coupling between the spin and lattice degrees of freedom has been the subject of intense investigation for many geometrically frustrated magnets, including especially pyrochlore materials. [21-25] The lineshapes of phonons and their renormalization at the onset of magnetic order yields specific information on the spinphonon coupling, and on the coupling between phonons and charge fluctuations in the case of electrically con- 

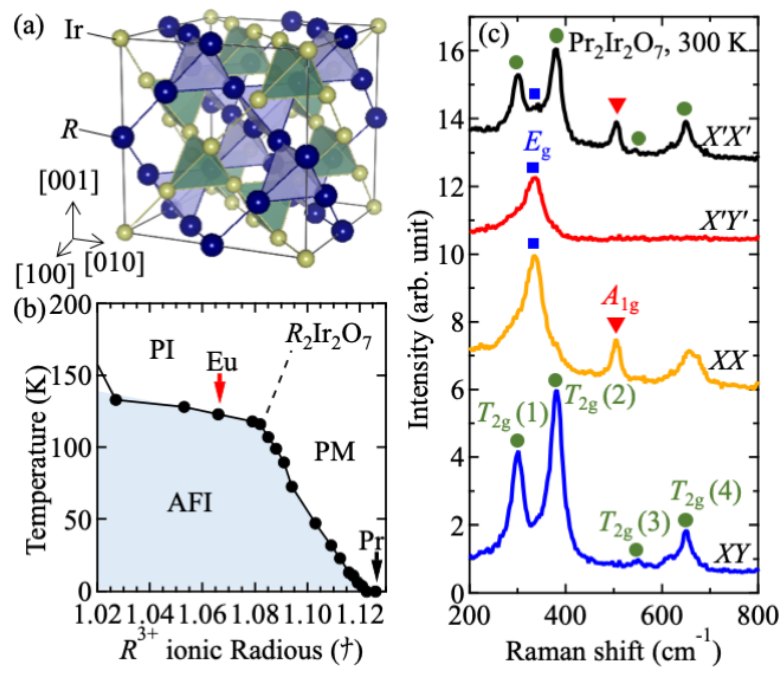

FIG. 1: (color online) (a) Schematic of the $R_{2} \operatorname{Ir}_{2} \mathrm{O}_{7}$ unit cell.(b) Phase diagram of $R_{2} \mathrm{Ir}_{2} \mathrm{O}_{7}$ as a function of rare-earth ionic radius and temperature. PI stands for paramagnetic insulator, PM stands for paramagnetic metal, and AFI stands for antiferromagnetic insulator. The $R=\mathrm{Eu}$ and $R=\operatorname{Pr}$ compounds are marked by arrows. (c) Raman scattering spectra of $\operatorname{Pr}_{2} \operatorname{Ir}_{2} \mathrm{O}_{7}$ for the polarization configurations $z(X X) \bar{z}$, $z(X Y) \bar{z}, z\left(X^{\prime} X^{\prime}\right) \bar{z}$, and $z\left(X^{\prime} Y^{\prime}\right) \bar{z}$ at room temperature. The red triangle indicates the $A_{1 g}$ mode, the blue squares are the $E_{g}$ mode, and the green circles indicate the $T_{2 g}$ modes.

ducting systems. We indeed find large phonon anomalies induced by the onset of magnetic order in $\mathrm{Eu}_{2} \mathrm{Ir}_{2} \mathrm{O}_{7}$, and discuss them in terms of different electron-phonon coupling mechanisms. In addition, we have observed magnon excitations in the AIAO state, which are consistent with recent observations by resonant inelastic x-ray scattering (RIXS). [27]

High-quality single crystals of $\mathrm{Pr}_{2} \mathrm{Ir}_{2} \mathrm{O}_{7}$ and $\mathrm{Eu}_{2} \mathrm{Ir}_{2} \mathrm{O}_{7}$ were grown by the KF flux method, as described previously. [26] In the as-grown state, the crystals are octahedron-shaped with (111) facets. We polished a (001) plane of the samples with lapping films of sufficiently fine grain-size for a contamination-free polarization analysis. The Raman experiments were carried out with a Jobin-Yvon LabRam HR800 spectrometer using the $632.8 \mathrm{~nm}$ excitation line of a He-Ne laser. Laser heating was minimized by keeping the laser power below 1.2 $\mathrm{mW}$, and all measured spectra were corrected for heating following Ref. 28. Specifically, systematic measurements of the laser power dependence of phonon energies showed that the heating occurs above $0.7 \mathrm{~mW}$. The laser power used in the present study may heat the sample by $\sim 20 \mathrm{~K}$. As we will show later, the corrected critical temperature, at which the spectra change remarkably, is consistent with the magnetic transition temperature.

The samples were placed in a He-flow cryostat, and the measurements were conducted in backscattering geometry with light propagating along the crystalline [001] axis, while the polarization of the incident and scattered light was varied within the (001) plane. The spectra were measured in four polarization configurations, namely $z(X X) \bar{z}, z(X Y) \bar{z}, z\left(X^{\prime} X^{\prime}\right) \bar{z}$, and $z\left(X^{\prime} Y^{\prime}\right) \bar{z}$, where $z(\bar{z})$ denote the propagation direction of the incident (scattered) light. $X(Y)$ represents the polarization of incident or scattered light along the [100] ([010]) crystallographic direction, and $X^{\prime}=X+Y, Y^{\prime}=X-Y$. According to a group-theoretical analysis of the pyrochlore structure (space group $F d \overline{3} m$ ), the corresponding Raman spectra for the four polarization configurations are composed of $A_{1 g}+E_{g}, T_{2 g}, A_{1 g}+E_{g}+T_{2 g}$, and $E_{g}$ contributions, respectively. The unit cell of $R_{2} \mathrm{Ir}_{2} \mathrm{O}_{7}$ consists of eight formula units. The factor group analysis yields the following optical phonon modes [29, 30]:

$$
\Gamma^{\mathrm{opt}}=A_{1 g}+E_{g}+4 T_{2 g}+7 T_{1 u} .
$$

The first six modes are Raman-active, and the last seven are infrared-active.

Figure 1(c) shows polarization-resolved Raman spectra of the $R=\operatorname{Pr}$ compound at room temperature. In the $z\left(X^{\prime} X^{\prime}\right) \bar{z}$ geometry, in which all Raman-active lattice vibration modes are allowed, we indeed observe the six peaks expected in the $F d \overline{3} m$ symmetry. In the $z\left(X^{\prime} Y^{\prime}\right) \bar{z}$ geometry, the expected $E_{g}$ mode is clearly visible around $330 \mathrm{~cm}^{-1}$. In the $z(X X) \bar{z}$ geometry, which allows an $A_{1 g}$ mode in addition to the $E_{g}$ mode, a sharp peak is observed at $508 \mathrm{~cm}^{-1}$ and can be assigned to the $A_{1 g}$ mode. Finally, four $T_{2 g}$ peaks are observed in the $z(X Y) \bar{z}$ scattering geometry at $302,378,554$, and $646 \mathrm{~cm}^{-1}$ (hereafter referred to as $T_{2 g}(1),(2),(3)$, and (4), respectively), in agreement with the group theoretical analysis. These modes are closely similar to phonon modes previously revealed in Raman experiments on isostructural pyrochlore oxides [30, 31].

To associate the observed modes with specific vibration patterns, we calculated the energy of zone-center phonon modes by density functional perturbation theory [32] as implemented in the Quantum ESPRESSO [33] package, with the lattice parameters reported in Ref. 26. The calculations were performed within the generalized gradient approximation of Perdew, Burke and Ernzerhof (PBE GGA) [34] using the fully-relativistic ONCV pseudopotentials [35]. We used a $4 \times 4 \times 4 k$-point grid for the Brillouin-zone integration and a plane-wave cutoff of $75 \mathrm{Ry}$. The results show that the $A_{1 g}$ and $E_{g}$ modes are mostly composed of Ir-O bond bending modes. The two $T_{2 g}$ peaks with high frequencies correspond to Ir-O stretching modes, while the lower two involve $R$-O stretching modes. Table 1 displays the observed and calculated phonon frequencies for $R=\mathrm{Pr}$ and $\mathrm{Eu}$. Most 
TABLE I: Observed and calculated phonon frequencies in $R_{2} \operatorname{Ir}_{2} \mathrm{O}_{7}$ at room temperature

\begin{tabular}{lcccccc}
\hline Material & \multicolumn{5}{c}{ observed/calculated frequencies } & $\left(\mathrm{cm}^{-1}\right)$ \\
& $T_{2 \mathrm{~g}}(1)$ & $E_{\mathrm{g}}$ & $T_{2 \mathrm{~g}}(2)$ & $A_{1 \mathrm{~g}}$ & $T_{2 \mathrm{~g}}(3)$ & $T_{2 \mathrm{~g}}(4)$ \\
\hline $\mathrm{Pr}_{2} \mathrm{Ir}_{2} \mathrm{O}_{7}$ & $301.8 / 300.0$ & $333.3 / 355.5$ & $377.9 / 400.9$ & $506.0 / 514.2$ & $554.3 / 567.1$ & $646.2 / 635.3$ \\
\hline $\mathrm{Eu}_{2} \mathrm{Ir}_{2} \mathrm{O}_{7}$ & $302.5 / 317.4$ & $336.8 / 371.2$ & $379.5 / 416.9$ & $507.8 / 529.6$ & $543.1 / 588.0$ & $680.2 / 676.8$ \\
\hline
\end{tabular}

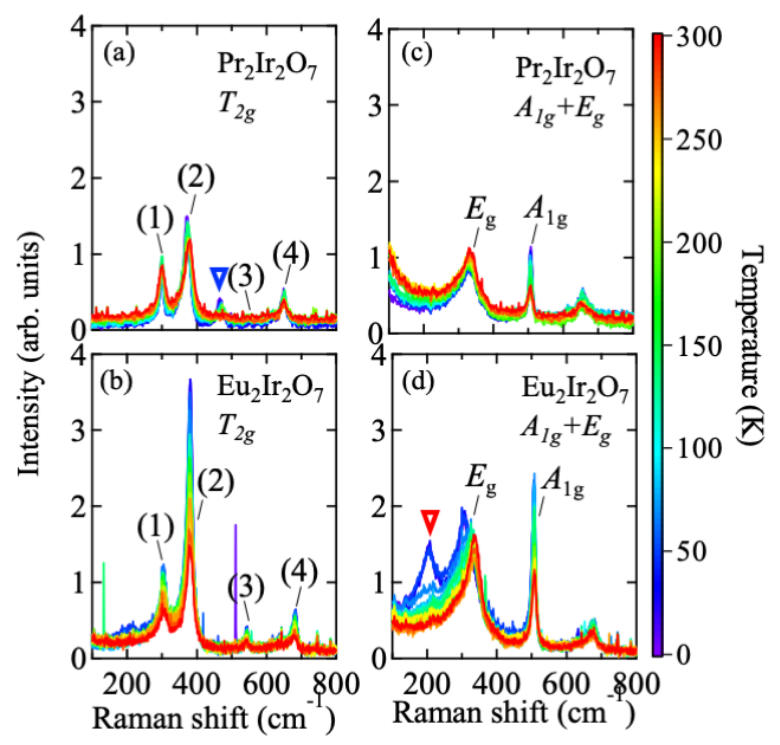

FIG. 2: (color online). Raman spectra of $(\mathrm{a}, \mathrm{b}) T_{2 g}$ modes and (c, d) $A_{1 g}+E_{g}$ modes for (a, c) $R=\operatorname{Pr}$ and (b ,d) $R=\mathrm{Eu}$ at several temperatures. The color bar represents the sample temperature.

of the peak energies are almost identical in the two compounds, except for the high-energy Ir-O stretching vibration with $T_{2 g}$ symmetry. The calculated and observed Raman energies are in good agreement, except for the $R$-ion dependence which is overestimated in the calculations. This discrepancy may in part be attributable to coupling between phonons and low-energy excitations of the valence electrons, which is not included here. It is noteworthy that the $E_{g}$ vibration mode is two-times broader than the other modes, as previously reported for the spinel chromite $\mathrm{CdCr}_{2} \mathrm{O}_{4}$ which exhibits a strong magnetoelastic coupling [36]. The same behavior is also observed in $R=\mathrm{Eu}$ (Fig. 2), despite the different ground states of both compounds.

We now turn to the temperature dependence of the Raman spectra for $R=\operatorname{Pr}$ and Eu (Fig. 2). Most of the phonon modes gradually sharpen with decreasing temperature, as expected as a consequence of lattice anharmonicity [37, 38]. For $\mathrm{Pr}_{2} \operatorname{Ir}_{2} \mathrm{O}_{7}$ we note a weak additional mode that appears at $450 \mathrm{~cm}^{-1}$ (marked by a blue antitriangle in Fig. 2(a)) at low temperature. Prior Raman and neutron scattering work on Pr pyrochlore compounds including $\mathrm{Pr}_{2} \mathrm{Zr}_{2} \mathrm{O}_{7}$, [39] $\mathrm{Pr}_{2} \mathrm{Ru}_{2} \mathrm{O}_{7}$ [40] and $\mathrm{Pr}_{2} \mathrm{Sn}_{2} \mathrm{O}_{7}$ [41] also detected a mode at this energy, which was attributed to Pr crystal-field excitations. The strongly reduced intensity of this mode at elevated temperatures may then reflect scattering of conduction electrons from the localized $\operatorname{Pr} 4 f$ electrons, as observed in other lanthanide compounds [42]. Additional Pr crystal-field excitations are expected at lower energies $\left(\sim 135 \mathrm{~cm}^{-1}\right)$, but these excitations are masked in our metallic compound by intense quasielastic scattering from low-energy charge excitations of conduction electrons (Fig. 2(c)).

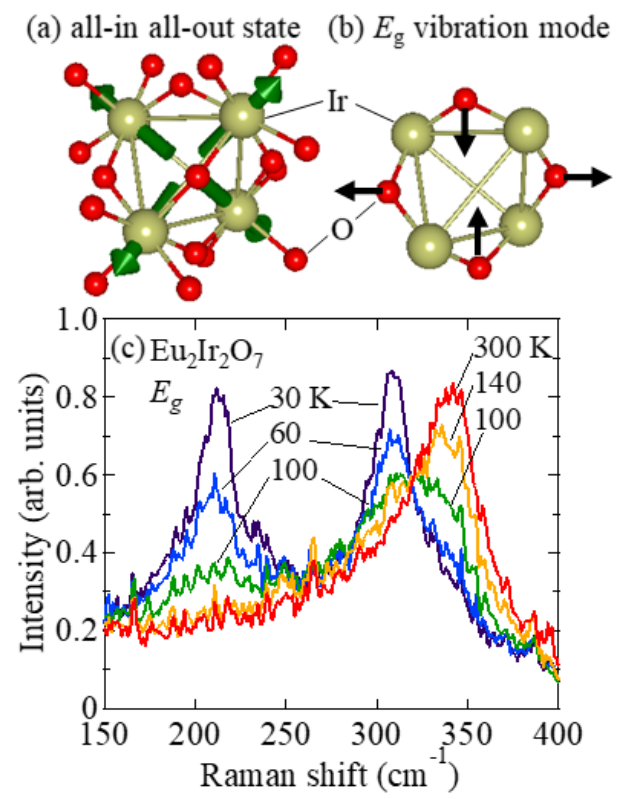

FIG. 3: (color online). (a) Schematic of the all-in/all-out magnetic ordering configuration. The arrows stand for the Ir magnetic moments. (b) Atomic displacements of the $E_{g}$ lattice vibration mode. The arrows indicate the motion of the $\mathrm{O}$ ions. (c) Raman spectra in the $z\left(X^{\prime} Y^{\prime}\right) \bar{z}$ scattering geometry for the $R=$ Eu compound at several temperatures.

The Raman spectra of the $R=$ Eu compound exhibit a more pronounced temperature dependence (Fig. 2(d)). Upon cooling, an intense peak emerges in the $E_{g}$ channel around $210 \mathrm{~cm}^{-1}$ (marked by a red antitriangle), and 

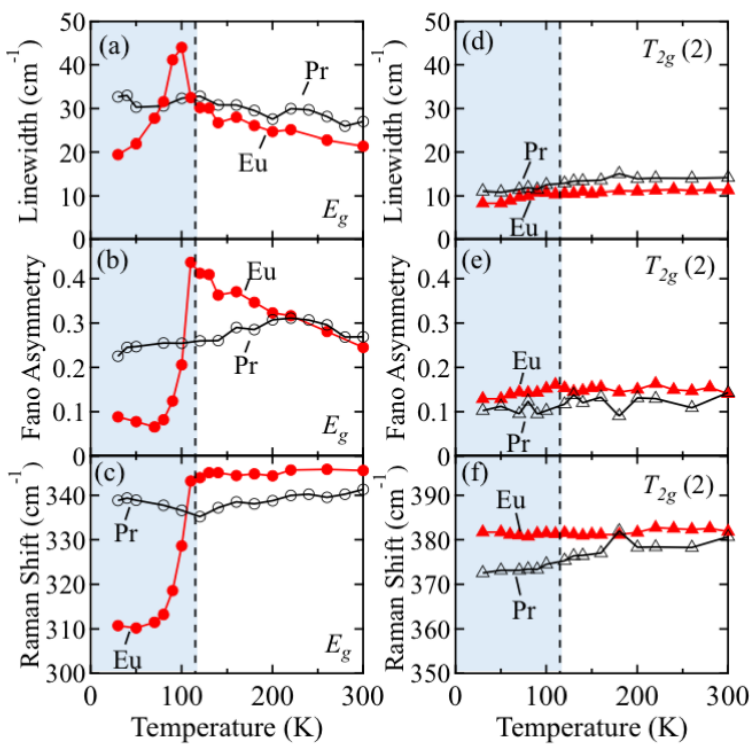

FIG. 4: (color online). Temperature dependence of (a),(d) Fano asymmetry, (b),(e) Raman shift, and (c),(f) linewidth of selected phonons in $\mathrm{Eu}_{2} \mathrm{Ir}_{2} \mathrm{O}_{7}$ and $\mathrm{Pr}_{2} \mathrm{Ir}_{2} \mathrm{O}_{7}$. Panels (a)-(c) are for the $T_{2 g}(2)$ phonon mode, and (d)-(f) for the $E_{g}$ mode, respectively.

the $E_{g}$ vibration mode at higher energy shows a strong anomaly. To gain more insight into this behavior, we conducted more detailed measurements of the temperature dependence of the $R=$ Eu phonons in the $z\left(X^{\prime} Y^{\prime}\right) \bar{z}$ configuration, which only allows $E_{g}$ symmetric excitations. Figure 3 displays the Raman spectra of $\mathrm{Eu}_{2} \mathrm{Ir}_{2} \mathrm{O}_{7}$ in the energy range from 150 to $400 \mathrm{~cm}^{-1}$. The new mode appears upon cooling below $T \sim T_{N}$ and hardens from 207 $\mathrm{cm}^{-1}$ at $100 \mathrm{~K}$ to $211 \mathrm{~cm}^{-1}$ at $30 \mathrm{~K}$, while the intensity increases in a manner consistent with the magnetic order parameter. We note that earlier work on $\mathrm{Eu}_{2} \mathrm{Ir}_{2} \mathrm{O}_{7}$ polycrystals also reported the appearance of three new peaks below $T_{\mathrm{N}}$ [43]. The frequency of one of these peaks is close to the one we observe, although the intensity is much smaller than ours. The other two peaks are not observed in our study, suggesting that they are related to the polycrystalline structure of the samples investigated earlier.

Following the discussion for $R=\operatorname{Pr}$ above, one might at first be tempted to assign the new peak to a Eu crystal-field excitation, although its intensity is much larger than the $450 \mathrm{~cm}^{-1}$ mode in $\operatorname{Pr}_{2} \mathrm{Ir}_{2} \mathrm{O}_{7}$. However, prior work on other Eu pyrochlore compounds has shown that the $\mathrm{Eu}^{3+}$ ions are in a singlet ground state, and that crystal-field excitations appear only at much higher energies. [44] Based on its appearance below $T_{N}$, we rather assign this mode to a single-magnon excitation that becomes Raman-active by virtue of the strong SOC and consequent strong single-ion anisotropy in the iridates. The intensity of the mode is higher than in typical collinear antiferromagnets, [45] but comparable to the one for magnons in other non-collinear magnets such as the canted antiferromagnets $\mathrm{FeBr}_{2}$ [46] and $\mathrm{FeF}_{2}$ [47]. The energy of the mode for $T \ll T_{N}$ is consistent with the zone-center gap of dispersive magnons recently observed by resonant inelastic x-ray scattering (RIXS). [27]. Close to $T_{N}$, the RIXS data indicate a stronger softening of the magnon than our Raman data, but both data sets are compatible within the combined error bars.

In view of the asymmetric lineshape of the observed phonons, we fitted them with Fano profiles written as $I(\omega)=I_{0}(q+\epsilon)^{2} /\left(1+\epsilon^{2}\right)$, where $\epsilon=\left(\omega-\omega_{0}\right) / \Gamma, \omega_{0}$ is the resonant energy, $\Gamma$ is the linewidth of the vibrational state, and $q$ is the Fano asymmetry parameter [48] which characterizes the strength of the coupling between the phonon and an excitation continuum. Figure 4 displays the temperature dependence of the resulting fitting parameters. Whereas the parameters characterizing the phonons in $\mathrm{Pr}_{2} \mathrm{Ir}_{2} \mathrm{O}_{7}$ and the $T_{2 g}$ modes in $\mathrm{Eu}_{2} \mathrm{Ir}_{2} \mathrm{O}_{7}$ exhibit smooth temperature dependences without any discernible anomalies (Fig. 4(d)-(f)), the $E_{g}$ lattice vibration mode in $\mathrm{Eu}_{2} \mathrm{Ir}_{2} \mathrm{O}_{7}$ is highly anomalous (Fig. 4(a)(c)). The phonon energy is nearly temperature independent in the paramagnetic state, but abruptly decreases by $\sim 35 \mathrm{~cm}^{-1}$ below $T_{N}$. In contrast to the other phonons, the linewidth of the $E_{g}$ mode increases with decreasing temperature in the paramagnetic state, and exhibits a sharp maximum below $T_{N}$ while the Fano asymmetry drops by a factor of four.

We first address the large softening of the $E_{g}$ phonon. Phonon energy shifts can in principle be caused by modulations of the static lattice structure across the magnetic transition. Since the specific heat indicates that the transition is continuous [4], the modulated lattice structure should be described by a subgroup of $F d \overline{3} \mathrm{~m}$. Continuous structural transitions have indeed been observed in other geometrically frustrated magnets with $F d \overline{3} m$ symmetry. For instance, the pyrochlore-type $\mathrm{Cd}_{2} \mathrm{Re}_{2} \mathrm{O}_{7}$ undergoes a transition from $F d \overline{3} m$ to $I 4_{1} /$ amd which is inversion asymmetric, $[22,23]$ and the spinel-type $(\mathrm{Cd}, \mathrm{Zn}) \mathrm{Cr}_{2} \mathrm{X}_{4}$ $(X=\mathrm{O}, \mathrm{S}, \mathrm{Se})$ exhibits a $F d \overline{3} m$-to- $F \overline{4} 3 m$ transition via a tetragonal distortion $[24,25,36]$. In each case, phonon peak splittings were reported not only for the $E_{g}$ symmetry, but also for modes in other scattering geometries, in contrast to our data which reveal appreciable anomalies only in $E_{g}$ symmetry. Furthermore, previous x-ray and neutron diffraction studies of $\mathrm{Eu}_{2} \mathrm{Ir}_{2} \mathrm{O}_{7}$ and other pyrochlore iridates report no indication of a lowering of the lattice symmetry $[16,18,51]$.

Frequency shifts of optical modes can also occur as a consequence of magnetostrictive effects following the Grüneisen law, as observed for instance in multiferroic $R \mathrm{CrO}_{3}$ which shows an abrupt lattice volume modification below the magnetic transition temperature $[49,50]$. 
In the case of $\mathrm{Eu}_{2} \mathrm{Ir}_{2} \mathrm{O}_{7}$, however, there is almost no anomaly in the temperature dependence of the lattice constants [51]. Finally, we note that Liang et al. discovered a $C_{4}$ symmetry breaking in the $R=\mathrm{Eu}$ compound by using torque magnetometry [52], which was later attributed to multipolar ordering [53]. However, in view of the absence of appreciable modifications of the atomic positions seen in the diffraction studies, it seems unlikely that this effect alone is responsible for the phonon anomalies we observed.

The large shift of the $E_{g}$ phonon energy at $T_{N}$ is therefore difficult to explain on the basis of static atomic displacements alone. This conclusion is underscored by the observation of the pronounced Fano lineshape of this mode, which indicates dynamical coupling of the vibrational mode to a continuum of excitations, and by the large anomalies of the parameters $\Gamma$ and $q$ at $T_{N}$. We distinguish three different mechanisms that can lead to such anomalies:

(i) Modulation of the exchange interactions via excitation of phonons. This "spin-phonon coupling" mechanism [54, 55] has been widely discussed for insulating compounds with $3 d$ valence electrons. [36, 56-60] The linewidth anomalies at the magnetic ordering transition generated by this mechanism are rather small in conventional magnetic insulators, but can become substantial if there are pronounced intersite spin-orbital fluctuations in the paramagnetic state,[61] which may well be the case in $\mathrm{Eu}_{2} \mathrm{Ir}_{2} \mathrm{O}_{7}$. We also note that the $E_{g}$ mode corresponds to an Ir-O-Ir bending mode (Fig. 3b), which is expected to modulate the superexchange interaction or Dzyaloshinskii-Moriya interaction between Ir pseudospins. These interactions can play an important role in the stabilization of the all-in all-out magnetic order, as suggested in Ref. [62].

(ii) Phonon-induced modulation of the crystal field, which affects magnetism via the on-site spin-orbit coupling. This mechanism has been discussed for $\mathrm{Cd}_{2} \mathrm{Os}_{2} \mathrm{O}_{7}$ [63] (where phonon anomalies of magnitude comparable to those in $\mathrm{Eu}_{2} \mathrm{Ir}_{2} \mathrm{O}_{7}$ have been reported) and for the insulating square-lattice iridates [28]. In iridates with the orbitally non-degenerate $J=\frac{1}{2}$ ground state, including $\mathrm{Eu}_{2} \mathrm{Ir}_{2} \mathrm{O}_{7}$, dynamical admixture of the $J=\frac{3}{2}$ levels can generate substantial pseudospin-phonon coupling and phonon linewidth anomalies. [28, 64]

(iii) Electron-phonon coupling through charge fluctuations. This effect can lead to large phonon lineshape anomalies at spin density wave transitions in metals which open up gaps on the Fermi surface, as observed for instance in the iron pnictides [65]. $\mathrm{Eu}_{2} \mathrm{Ir}_{2} \mathrm{O}_{7}$ exhibits an optical gap of $\sim 200 \mathrm{meV}$, much larger than the phonon energy, but the gap is soft and shows a long low-energy tail. [6] The electrical conductivity of $\mathrm{Eu}_{2} \mathrm{Ir}_{2} \mathrm{O}_{7}$ decreases with decreasing temperature, but its magnitude is substantial in the paramagnetic state and decreases sharply upon cooling below $T_{N}$. Temperature dependent charge fluctuations may therefore also contribute to the phonon anomalies in this material.

These considerations suggest that the Ir-O-Ir bondbending vibration in $\mathrm{Eu}_{2} \mathrm{Ir}_{2} \mathrm{O}_{7}$ is strongly coupled to a continuum composed of spin, charge, and orbital excitations via a confluence of all three mechanisms. With the onset of all-in/all-out magnetic order, a gap (or pseudogap) of magnitude larger than the phonon energy develops in this continuum, so that the coupling is quenched and the Fano asymmetry is reduced (Fig. 4(b)). Since electronic spectral weight is expected to pile up at energies above the gap, the non-monotonic $T$-dependence of the linewidth $\Gamma$ may be a consequence of the continuous closure of the gap upon approaching $T_{N}$. An analogous temperature evolution of $\Gamma$ is also observed for some phonons in high-temperature superconductors due to the opening of the superconducting gap. [66]

In conclusion, our Raman experiments on $\mathrm{Eu}_{2} \mathrm{Ir}_{2} \mathrm{O}_{7}$ have revealed unusually strong anomalies of a specific phonon mode that involves Ir-O-Ir bond bending vibrations. These data should motivate in-depth theoretical work on the microscopic mechanism responsible for the electron-phonon coupling, and on the influence of electron-phonon interactions on the anomalous electronic properties and phase behavior of the pyrochlore iridates and related compounds.

We are grateful to Hakuto Suzuki and Giniyat Khaliullin for fruitful discussions. This work was supported by German Research Foundation (DFG) under grant TRR80, by the Japan Society for the Promotion of Science through the Funding Program for World-Leading Innovative R\&D on Science and Technology (FIRST Program) on 'Quantum Science on Strong Correlation' initiated by the Council for Science and Technology Policy, by JSPS Grant-in-Aid for Scientific Research (No. 26103006, No. 24224009, 18H04214, and 16H00981), and by PRESTO (No. JPMJPR15R5) and CREST (No. JPMJCR16F1 and JPMJCR1874), Japan Science and Technology Japan.

[1] B. Keimer and J.E. Moore, Nat. Phys. 13, 1045 (2017).

[2] B. J. Kim et al., Phys. Rev. Lett. 101, 076402 (2008).

[3] W. Witczak-Krempa, G. Chen, Y.B. Kim, L. Balents, Annu. Rev. Condens. 5, 57 (2014).

[4] K. Matsuhira, M. Wakeshima, Y. Hinatsu, and S. Takagi, J. Phys. Soc. Jpn. 80, 094701 (2011).

[5] K. Ueda et al., Phys. Rev. Lett. 109, 136402 (2012).

[6] K. Ueda, J. Fujioka, and Y. Tokura, Phys. Rev. B 93, 245120 (2016).

[7] M. Sakata et al., Phys. Rev. B 83, 041102 (2011).

[8] F. F. Tafti, J. J. Ishikawa, A. McCollam, S. Nakatsuji, and S. R. Julian, Phys. Rev. B 85, 205104 (2012).

[9] K. Ueda, J. Fujioka, C. Terakura, and Y. Tokura, Phys. Rev. B 92, 121110 (2015).

[10] K. Ueda, J. Fujioka, B.-J. Yang, J. Shiogai, A. Tsukazaki, 
S. Nakamura, S. Awaji, N. Nagaosa, and Y. Tokura, Phys. Rev. Lett. 115, 056402 (2015).

[11] Z. Tian et al., Nat. Phys. 12, 134 (2016).

[12] K. Ueda, T. Oh, B.-J. Yang, R. Kaneko, J. Fujioka, N. Nagaosa, and Y. Tokura, Nat. Commun. 8, 15515 (2017).

[13] K. Ueda, R. Kaneko, H. Ishizuka, J. Fujioka, N. Nagaosa, and Y. Tokura, Nat. Commun. 9, 3032 (2018).

[14] S. Nakatsuji, Y. Machida, Y. Maeno, T. Tayama, T. Sakakibara, J. van Duijn, L. Balicas, J. N. Millican, R. T. Macaluso, and Julia Y. Chan, Phys. Rev. Lett. 96, 087204 (2006).

[15] T. Kondo et al., Nat. Commun. 6, 10042 (2015).

[16] H. Sagayama, D. Uematsu, T. Arima, K. Sugimoto, J. J. Ishikawa, E. O'Farrell, and S. Nakatsuji, Phys. Rev. B 87, 100403 (2013).

[17] K. Tomiyasu, K. Matsuhira, K. Iwasa, M. Watahiki, S. Takagi, M. Wakeshima, Y. Hinatsu, M. Yokoyama, K. Ohoyama, and K. Yamada, J. Phys. Soc. Jpn. 81, 034709 (2012).

[18] H. Guo, C. Ritter, and A. C. Komarek, Phys. Rev. B 94, 161102(R) (2016).

[19] M. Imada, A. Fujimori, and Y. Tokura, Rev. Mod. Phys. 70, 1039 (1998).

[20] B.-J. Yang and Y. B. Kim, Phys. Rev. B 82, 085111 (2010).

[21] J. S. Gardner, M. J. P. Gingras, and J. E. Greedan, Rev. Mod. Phys. 82, 53 (2010).

[22] C. S. Knee et al., Phys. Rev. B 71, 214518 (2005).

[23] J.-I. Yamaura, K. Takeda, Y. Ikeda, N. Hirao, Y. Ohishi, T. C. Kobayashi, and Z. Hiroi, Phys. Rev. B 95, 020102 (2017).

[24] A. B. Sushkov, O. Tchernyshyov, W. Ratsliff II, S. W Cheong, and H. D. Drew, Phys. Rev. Lett. 94, 137202 (2005).

[25] V. Gnezdilov, P. Lemmens, Y. G. Pashkevich, Ch. Payen, K. Y. Choi, J. Hemberger, A. Loidl, and V. Tsurkan, Phys. Rev. B 84, 045106 (2011).

[26] J. N. Millican et al., Mater. Res. Bull. 42, 928 (2007).

[27] S. H. Chun et al., Phys. Rev. Lett. 120, 177203 (2018).

[28] H. Gretarsson, N. H. Sung, M. Höppner, B. J. Kim, B. Keimer, and M. Le Tacon, Phys. Rev. Lett. 116, 136401 (2016).

[29] H. C. Gupta, S. Brown, N. Rani, and V. B. Gohel, J. Raman Spectrosc. 32, 41 (2001).

[30] M. T. Vandenborre, E. Husson, J. P. Chatry, and D. Michel, J. Raman Spectrosc. 14, 63 (1983).

[31] K. Taniguchi, T. Katsufuji, S. Iguchi, Y. Taguchi, H. Takagi, and Y. Tokura, Phys. Rev. B 70, 100401 (2004).

[32] S. Baroni, S. de Gironcoli, A. Dal Corso, and P. Giannozzi, Rev. Mod. Phys. 73, 515 (2001).

[33] P. Giannozzi, S. Baroni, N. Bonini, M. Calandra, R. Car, C. Cavazzoni, D. Ceresoli, G. L. Chiarotti, M. Cococcioni, I. Dabo et al., J. Phys.: Condens. Matter 21, 395502 (2009).

[34] J. P. Perdew, K. Burke, and M. Ernzerhof, Phys. Rev. Lett. 77, 3865 (1996).

[35] P. Scherpelz, M. Govoni, I. Hamada, G. Galli, J. Chem. Theory Comput. 12, 3523 (2016).

[36] Ch. Kant et al., Phys. Rev. B 80, 214417 (2009).

[37] P. G. Klemens, Phys. Rev. 148, 845 (1966).

[38] M. Balkanski, R. F. Wallis, and E. Haro, Phys. Rev. B
28, 1928 (1983).

[39] K. Kimura, S. Nakatsuji, J.-J. Wen, C. Broholm, M.B. Stone, E. Nishibori, and H. Sawa, Nat. Comm. 4, 1934 (2013).

[40] J. van Duijn et al., Phys. Rev. B 96, 094409 (2017).

[41] S. Saha, S. Prusty, S. Singh, R. Suryanarayanan, A. Revcolevschi, and A.K. Sood, J. Sol. State Chem. 184, 2204 (2011).

[42] R. Feile, M. Loewenhaupt, J. K. Kjems, and H. E. Hoenig, Phys. Rev. Lett. 47, 610 (1988)

[43] T. Hasegawa, N. Ogita, K. Matsuhira, S. Takagi, M. Wakeshima, Y. Hinatsu, and M. Udagawa, J. Phys.: Conf. Ser. 200, 012054 (2010).

[44] A. Nag, P. Dasgupta, Y.M. Jana, and D. Ghosh, J. Alloy Compd. 384, 6 (2004).

[45] W. Hayes and R. Loudon, Scattering of light by crystals (Dover Publications Inc., Mineola, New York).

[46] G. C. Psaltakis, G. Mischler, D. J. Lockwood, M. G. Cottam, A. Zwick, and S. Legrand, J. Phys. C: Solid State Phys., 17, 1735 (1984).

[47] D. J. Lockwood, M. G. Cottam, V. C. Y. So, and R. S. Katiyar, J. Phys. C: Solid State Phys. 17, 6009 (1984).

[48] U. Fano, Phys. Rev. 124, 1866 (1961).

[49] M. Udagawa, K. Kohn, N. Kashizuka, and T. Tsushima, Solid State Commun. 16, 779 (1975).

[50] V. S. Bhadram, B. Rajeswaran, A. Sundaresan, and C. Narayana, EPL 101, 17008 (2013).

[51] H. Takatsu, K. Watanabe, K. Goto, and H. Kadowaki, Phys. Rev. B 90, 235110 (2014).

[52] T. Liang, T. H. Hsieh, J. J. Ishikawa, S. Nakatsuji, L. Fu, and N. P. Ong, Nature Phys. 10, 1038 (2017).

[53] Y. Wang, H. Weng, L. Fu, and X. Dai, Phys. Rev. Lett. 119, 187203 (2017).

[54] W. Baltensperger, J. Appl. Phys. 41, 1052 (1970).

[55] N. Suzuki and H. Kamimura, J. Phys. Soc. Jpn. 35, 985 (1973).

[56] D. J. Lockwood and M. G. Cottam, J. Appl. Phys. 64, 5876 (1988).

[57] K. Wakamura and T. Arai, J. Appl. Phys. 63, 5824 (1988).

[58] E. Granado, N. O. Moreno, A. Garcia, J. A. Sanjurjo, C. Rettori, I. Torriani, S. B. Oseroff, J. J. Neumeier, M. J. McClellan, S.-W. Cheong, and Y. Tokura, Phys. Rev. B 58, 11435 (1998).

[59] E. Granado et al., Phys. Rev. B 60, 11879 (1999).

[60] J. Laverdière et al., Phys. Rev. B 73, 214301 (2006).

[61] C. Ulrich, G. Khaliullin, M. Guennou, H. Roth, T. Lorenz, and B. Keimer, Phys. Rev. Lett 115, 156403 (2015).

[62] Maged Elhajal, Benjamin Canals, Raimon Sunyer, and Claudine Lacroix, Phys. Rev. B 71, 094420 (2005).

[63] C. H. Sohn et al., Phys. Rev. Lett. 118, 117201 (2017).

[64] H. Gretarsson, J. Sauceda, N. H. Sung, Höppner, M. Minola, B. J. Kim, B. Keimer, and M. Le Tacon Phys. Rev. B 96, 115138 (2017).

[65] M. Rahlenbeck, G. L. Sun, D. L. Sun, C. T. Lin, B. Keimer, and C. Ulrich, Phys. Rev. B 80, 064509 (2009).

[66] M. Bakr, A. P. Schnyder, L. Klam, D. Manske, C. T. Lin, B. Keimer, M. Cardona, and C. Ulrich, Phys. Rev. B 80, 064505 (2009). 